\title{
Need for Development of Paediatric Rheumatology Curriculum in Paediatrics Teaching Worldwide -Lessons Being Learnt from SHARE (A Single Hub and Access Point from Paediatric Rheumatology in Europe)
}

\section{Kulvinder Kochar Kaur*}

Scientific Director, Dr Kulvinder Kaur Centre for Human Reproduction, Jalandhar, Punjab, India

*Corresponding Author: Kulvinder Kochar Kaur, Scientific Director, Dr Kulvinder Kaur Centre for Human Reproduction, Jalandhar, Punjab, India.

Received: July 29, 2019; Published: August 13, 2019

Innovative research in childhood rheumatic diseases mandates international collaborations. However, researchers struggle with significant regulatory heterogeneity; an enabling European Union(EU)-wide framework is missing. Thus a systematic review for the best practice and to establish recommendations for collaborative research was carried out by Kremmerie-Deschner., et al. in 2018 The Paediatric Rheumatology European Single Hub and Access point for Paediatric Rheumatology In Europe (SHARE) project enabled a scoping review and expert discussion, which then informed the systematic literature review. Published evidence was synthesized, recommendations were drafted. An iterate review process and consultations with Ethics Committees and European experts for ethical and legal aspects of pediatric research refined the recommendations. SHARE experts and patient representatives vetted the proposed recommendations at a consensus meeting using Nominal Group Technique. Agreement of $80 \%$ was mandatory for inclusion. The systematic literature review returned 1319 records. A total of 223 full text publications plus 22 international normative documents were reviewed ;85 publications and 16 normative documents were included. A total of 21 principle ( 8 and 9), consent to paediatric research (10-14), paediatric data bank and biobank (15 and 16), sharing of data and samples (17-19), and commercialization and third parties (20 and 21). The refined recommendations resulted in an agreement of $>80 \%$ of all recommendations. The SHARE initiative established the first recommendations for Paediatric Rheumatology collaborative research across borders in Europe. These provide strong support for an urgently needed European framework and evidence based guidance for implementation. Such changes will promote research in children with rheumatic diseases [1].
Information technology in Paediatric Rheumatology has seen several exciting developments in recent years. The new multidimensional questionnaires for juvenile idiopathic arthritis, juvenile dermatomyositis, and juvenile autoinflammatory diseases integrate all major parent and child related outcomes(PCROs) used in these diseases into a single tool, and provide an effective guide to manage, document change in health,assess effectiveness and safety of the medications currently used in juvenile idiopathic arthritis, particularly biological agents, through collection of prospective data in a large, multinational sample of patient. Children and their parents are directly involved in the data collection by means of the regular completion of digital version of a multidimensional questionnaires. The Patient-Reported Outcomes Measurement Information System (PROMIS) employs modern measurement science to advance assessment of PCRO's particularly HRQL and offers multidimensional profile measures. The conceptual link of paediatric PROMIS with adult instruments facilitates harmonization of assessments made in children and adolescents with those carried out in young adults in the process of transition of medical care. Development of electronic versions of questionnaires that permit their completion through smartphones or touchscreen devices will revolutionize information collection from parents and children, foster the regular collection of PCRO's in routine care, and ultimately prove the quality of self reported health data, and patient outcomes [2].

The Paediatric Rheumatology European Society (PReS) has an overarching aim to promote Paediatric Rheumatology as a subspeciality across Europe and also further a field through the expanding international membership. The actions of the PReS ETC aim to improve access to, and provision high quality clinical care de- 
livered by an appropriately trained workforce and ultimately improve outcomes for children and families. The emergence of Basic and Advanced courses, specialist courses, online resources and the EMERGE (Emerging Rheumatolo Gists and rEsearchers) fellowship programme, supplement the annual scientific meeting of PReS and YIM (young investigators meeting) as a means to facilitate advances in knowledge being implemented into high quality evidence based clinical care. It is recognized that more work is required to enable and support the expanding Paediatric Rheumatology community both in Europe and Internationally. The uptake and implementation of the new 2016 European Syllabus into training programmes needs to address the accreditation of training centres, a proposed European Subspecialist examination and certification in Paediatric Rheumatology.

There is an overarching structure to the education and teaching activities of the PReS ETC to address the expanding needs of the broad Paediatric Rheumatology community with the content based on the 2016 European syllabus for training. The format and structure of the PReS educational portfolio, much of which is free and open to all, services as a template relevant to the wider international Paediatric Rheumatology. There is clear synergy between the educational and training activities of PReS and other initiatives led by Paediatric Rheumatology organizations elsewhere in the world (like those developed by ACR, CARRA, BSR, BSPAR, APRG). Through working with colleagues around the world the aim has been to "reach out", raise awareness and facilitate growth of Paediatric Rheumatology relevant to the local context. Thus with this aim, the current portfolio of PReS educational activities and their relevance to the international paediatric community had been the aim of this review by Foster., et al. [3]. Till date, PReS Basic courses have been held in countries where Paediatric Rheumatology is less well developed Mumbai (India 2012), Sao Paulo (Brazil 2015), Budapest (Hungary 2015) and Cape Town (South Africa 2017) with future courses planned in Ukraine and South East Asia [3].

\section{Bibliography}

1. Kremmerie-Deschner JB., et al. "Recommendations for collaborative paediatric research including biobanking in Europe: a single Hub and Access point for paediatric Rheumatology in Europe (SHARE) initiative". Annals of the Rheumatic Diseases 77.3 (2018): 319-327.
2. Consolaro A., et al. "Information technology in Paediatric Rheumatology". Clinical and Experimental Rheumatology 34 (2016): S11-S16.

3. Foster HE., et al. "Educational initiatives and training for Paediatric Rheumatology in Europe". Paediatric Rheumatology 16 (2018): 77.

Volume 2 Issue 9 September 2019 (C) All rights are reserved by Kulvinder Kochar Kaur. 